Revista de Metalurgia

Octubre-Diciembre 2019, 55(4), e153

ISSN-L: 0034-8570

https://doi.org/10.3989/revmetalm.153

\title{
Corrosión del aluminio 1050 en atmósferas costeras
}

\author{
Iván Díaz , María Fuentes ${ }^{\mathrm{a}}$, Daniel de la Fuente ${ }^{\mathrm{a}}$, Belén Chico ${ }^{\mathrm{a}}$, \\ José A. Jiménez ${ }^{\mathrm{a}}$, Manuel Morcillo ${ }^{\mathrm{a}, \bigotimes}$ \\ ${ }^{a}$ Centro Nacional de Investigaciones Metalúrgicas (CENIM, CSIC). Avda. Gregorio del Amo 8, 28040 Madrid, España \\ ( $\bowtie$ Autor para la correspondencia: morcillo@cenim.csic.es)
}

Enviado: 31 January 2019; Aceptado: 20 November 2019; Publicado on-Line: 20 December 2019

\begin{abstract}
RESUMEN: Existen escasos estudios realizados sobre la corrosión atmosférica del aluminio en zonas costeras en los que se considere un amplio intervalo de salinidades atmosféricas. El trabajo, que aquí se presenta, ofrece los resultados de una investigación llevada a cabo en seis estaciones de ensayo situadas en atmósferas marinas puras ubicadas a diferentes distancias de la línea de costa dentro del Parque eólico Cabo Vilano (Galicia). Se expusieron probetas de ensayo de aluminio 1050 en las atmósferas marinas durante 3, 6, 9 y 12 meses, determinándose la corrosión experimentada por el aluminio (formación de picaduras) en función del tiempo de exposición. Se analizó la naturaleza y composición de los productos de corrosión formados mediante difracción de rayos X con ángulo rasante. Asimismo, se estudió la morfología del ataque experimentado por el aluminio mediante microscopía electrónica de barrido con espectrometría de rayos X por dispersión de energías. Como resultados más relevantes cabe destacar: (a) El alcance de la velocidad de corrosión disminuye conforme nos alejamos de la línea de costa y avanza el tiempo de exposición, (b) El producto de corrosión formado es gibsita, (c) El progreso de las picaduras se debe a la presencia de los aniones $\mathrm{Cl}^{-} \mathrm{y} \mathrm{SO}_{4}^{2-}$, procedentes del aerosol marino que migran hacia el fondo de las picaduras.
\end{abstract}

PALABRAS CLAVE: Aluminio; Corrosión atmosférica; Corrosión por picaduras; Salinidad

Citar como/Citation: Díaz, I.; Fuentes, M.; de la Fuente, D.; Chico, B.; Jiménez, J.A.; Morcillo, M. (2019). “Corrosión del aluminio 1050 en atmósferas costeras". Rev. Metal. 55(4): e153. https://doi.org/10.3989/revmetalm.153

ABSTRACT: Corrosion of 1050 aluminium in coastal atmospheres. Very few studies on the atmospheric corrosion of aluminium in coastal areas have considered a wide range of atmospheric salinities. This paper reports the results of research carried out at six testing stations located in pure marine atmospheres at different distances from the shoreline at Cabo Vilano wind farm (Galicia). 1050 aluminium test specimens were exposed in the marine atmospheres for 3, 6, 9 and 12 months, determining the corrosion experienced by aluminium (pitting formation) as a function of exposure time. The nature and composition of the corrosion products formed was analysed by grazing incidence X-ray diffraction and the morphology of the attack experienced by the aluminium was studied by scanning electron microscopy with energy dispersive X-ray spectrometry. The most salient results include the following: (a) The corrosion rate decreases with distance from the shoreline and with exposure time, (b) The corrosion product formed is gibbsite, (c) The development of pitting is due to the presence of $\mathrm{Cl}^{-}$and $\mathrm{SO}_{4}{ }^{2-}$ anions from the marine aerosol that migrate to the bottom of the pits.

KEYWORDS: Aluminium; Atmospheric corrosion; Pitting corrosion; Salinity

ORCID ID: Iván Díaz (https://orcid.org/0000-0003-3981-985X); María Fuentes (https://orcid.org/0000-0002-3001-9808); Daniel de la Fuente (https://orcid.org/0000-0003-4540-3180); Belén Chico (https://orcid.org/0000-0001-8697-6298); José A. Jiménez (https://orcid.org/0000-0003-4272-6873); Manuel Morcillo (https://orcid.org/0000-0002-7355-0092)

Copyright: (C) 2019 CSIC. Este es un artículo de acceso abierto distribuido bajo los términos de la licencia de uso y distribución Creative Commons Reconocimiento 4.0 Internacional (CC BY 4.0). 


\section{INTRODUCCIÓN}

El aluminio, en competencia con los aceros, es uno de los metales más ampliamente utilizados, siendo aplicado abundantemente en sectores de la arquitectura (recubrimientos de fachadas, marcos de ventana, etc.), transporte, obras públicas, telecomunicaciones, electrónica, etc. Su buena apariencia, ligereza, fabricabilidad y buenas propiedades tanto físicas, mecánicas como de resistencia a la corrosión han hecho de este material una alternativa económica y atractiva para una amplia variedad de aplicaciones.

Cuando la superficie de aluminio entra en contacto con el aire, la oxidación directa forma espontáneamente una delgada película de óxido de aluminio $\left(\gamma-\mathrm{Al}_{2} \mathrm{O}_{3}\right)$, compacta, amorfa, tenaz e inerte (pasiva) que suministra gran protección al aluminio base. Según las condiciones de formación, el espesor de la película de alúmina varía entre 40 y $100 \AA$ A. Esta película se compone a su vez de dos capas (Vargel, 1981): una capa más compacta (capa barrera), próxima al aluminio base, de 20-25 ̊̊ de espesor, y otra capa más externa y porosa de bohemita $(\gamma-\mathrm{AlOOH})$ o bayerita $\left(\mathrm{Al}(\mathrm{OH})_{3}\right)$ cuyo espesor crece lentamente con la humedad de la atmósfera.

Aniones tales como $\mathrm{SO}_{4}^{2-}$, o $\mathrm{Cl}^{-}$, depositados sobre la superficie del óxido natural, reaccionan con él dando lugar a sales solubles (p. ej. $\mathrm{Al}_{2}\left(\mathrm{SO}_{4}\right)_{3}$, $\mathrm{AlCl}_{3}$, etc.), o bien se incorporan a la red del óxido formando una variedad de complejos y sales básicas (Kucera y Mattson, 1987). La película de óxido es permeable a los iones cloruro; éstos, debido a su elevada densidad de carga, migran con facilidad hacia el interior de la película, favoreciendo a su vez la migración de los cationes $\mathrm{Al}^{3+}$ hacia el exterior de la misma (Kaesche, 1974). Ello hace que los iones $\mathrm{Cl}^{-}$promuevan fácilmente el picado del aluminio.

La corrosión atmosférica del aluminio se manifiesta a través de dos morfologías principales: empañamiento (deslustrado, moteado, pérdida de brillo) $\mathrm{y}$ formación de picaduras. La primera no es un ataque propiamente dicho y precede a la formación de picaduras (Morcillo et al., 2000a; Morcillo et al., 2000b; Morcillo et al., 2000c). La formación de picaduras tiene lugar en zonas defectuosas de la película de óxido natural, tales como: adelgazamiento, rotura, presencia de vacantes, zonas de emergencia de dislocaciones, impurezas, elementos de aleación, etc. Por tanto, se establecen pilas locales en la superficie del aluminio causadas por áreas microscópicas anódicas muy activas en las que el metal contiguo actúa de cátodo. La reacción global anódica seguida de la hidrólisis del agua, Ec. (1):

$$
\mathrm{Al}+3 \mathrm{H}_{2} \mathrm{O} \rightarrow \mathrm{Al}(\mathrm{OH})_{3}+3 \mathrm{H}^{+}+3 \mathrm{e}^{-}
$$

supone la liberación de iones hidrógeno, cuya acumulación dentro de las picaduras provoca un descenso gradual del $\mathrm{pH}$ que favorece el continuo funcionamiento de la pila de corrosión (Otero et al., 1971).

Según Leygraf et al. (2016), la adsorción de iones cloruro sobre la capa acuosa, existente en la superficie del aluminio expuesto a la atmósfera marina, causa el adelgazamiento local de la película de óxido de aluminio o bien la formación de una picadura con la disolución simultanea de iones de aluminio. Posteriormente, tiene lugar la formación de distintos complejos transitorios que conducen a la formación de compuestos solubles como: hidróxicloruros de aluminio $\left(\mathrm{Al}(\mathrm{OH})_{2} \mathrm{Cl}, \mathrm{Al}(\mathrm{OH}) \mathrm{Cl}_{2}\right.$, etc. $)$ y cloruro de aluminio $\left(\mathrm{AlCl}_{3}\right)$, que actúan como potenciales precursores para la formación de $\mathrm{Al}(\mathrm{OH})_{3}$.

$$
\begin{aligned}
& \mathrm{Al}(\mathrm{OH})_{2} \mathrm{Cl}+\mathrm{Cl}^{-} \rightarrow \mathrm{Al}(\mathrm{OH}) \mathrm{Cl}_{2}+\mathrm{OH}^{-} \\
& \mathrm{Al}(\mathrm{OH}) \mathrm{Cl}_{2}+\mathrm{Cl}^{-} \rightarrow \mathrm{AlCl}_{3}+\mathrm{OH}^{-}
\end{aligned}
$$

El hidróxido de aluminio no es soluble y precipita en forma de una masa blanca gelatinosa que recubre la picadura a modo de pústulas voluminosas.

Se dispone de amplia información sobre la corrosión atmosférica del aluminio y de los mecanismos de formación de los productos de corrosión; desde los primeros estudios de Walton y King (1955), Booth y Dodard (1965), Berukshtis y Klark (1966), Carter (1968), McGeary et al. (1968), Mattsson y Lindgren (1968), Golubev y Kadyrov (1969), Speding (1971), Mikhailovskii et al. (1973), Haynie et al. (1976), Otero et al. (1978), Longo y Durmann (1978), Lashermes et al. (1982), y Graedel (1989), a otros relativamente más recientes de Morcillo et al., 2000a, Morcillo et al., 2000b, Morcillo et al., 2000c, González et al. (2002), Vera et al. (2006), Natesan et al. (2006), de la Fuente et al. (2007), Portella et al. (2012), Liu et al. (2014), Cai et al. (2018) y Liang et al. (2018).

Se han realizado, asimismo, notables esfuerzos para determinar la magnitud del ataque (pérdida de masa) y su evolución con el tiempo de exposición (Tidblad et al., 1998), habiéndose desarrollado diversos modelos para describir la influencia de los diferentes parámetros ambientales con respecto a la velocidad de corrosión atmosférica del aluminio, en particular con el contenido de $\mathrm{SO}_{2}$ en la atmósfera y la salinidad atmosférica. Ensayos de larga duración realizados en diferentes partes del mundo (Walton et al., 1953; Kentzler y Hoff, 1975) y en una diversidad de atmósferas han mostrado que la velocidad de corrosión del aluminio decrece muy rápidamente con el tiempo de exposición.

El aluminio de alta pureza presenta, por lo general, menos picaduras, aunque son más profundas que las del aluminio de menor pureza o aleado. Las 
mayores dimensiones de las picaduras parecen ser atribuibles a un menor número de puntos anódicos para una extensa área catódica disponible para la reducción del oxígeno; por el contrario, el aluminio aleado de menor pureza sufre un ataque más diseminado, pero menos profundo debido a que el número de puntos anódicos y el área anódica es mayor (Otero et al., 1971).

La experiencia ha demostrado que la velocidad de penetración de las picaduras que se forman sobre el aluminio en la atmósfera decrece con el tiempo (Walton et al., 1953; McGeary et al., 1968; Mattsson y Lindgren, 1968; Kentzler y Hoff, 1975). Aziz y Godard (1952) estimaron que la profundidad máxima de las picaduras (e) está relacionada con el tiempo $(\mathrm{t})$ de exposición mediante la fórmula:

$$
\mathrm{e}=\mathrm{Kt}^{1 / 2}
$$

donde $\mathrm{K}$ es una constante.

Este descenso en la profundidad máxima de las picaduras se debe a la existencia de $\mathrm{Al}(\mathrm{OH})_{3}$ que recubre la picadura y que ejerce un cierto efecto barrera. Se ha comprobado que la máxima profundidad de picadura después del primer año de exposición supone en muchos casos más del $80 \%$ de la máxima profundidad de picadura encontrada después de los primeros seis años de exposición (Carter, 1968).

\section{MATERIALES Y MÉTODOS}

\subsection{Probetas de ensayo}

Se prepararon probetas de dimensiones 100x 50x2 $\mathrm{mm}$ mediante corte por cizalla a partir de una chapa de Al 1050 H24 (99,55 \% Al). La composición química del aluminio se muestra en la Tabla 1.

Antes de su exposición a la atmósfera, se las sometió a un proceso de preparación superficial consistente en un lijado en seco con papel de carburo de silicio, desengrase con acetona y lavado con detergente, comprobando la calidad del desengrase por observación de la continuidad de una película acuosa depositada sobre la superficie. Posteriormente las probetas se sumergieron durante 15 min en una solución de etanol con agitación ultrasónica, secándose a continuación con aire caliente. Finalmente fueron pesadas hasta la décima de miligramo en una microbalanza Mettler AT 261 Delta Range y almacenadas en un desecador que contenía gel de sílice.

\subsection{Exposición atmosférica}

Los parámetros ambientales que han caracterizado a la zona experimental durante el desarrollo del estudio, abril 2017-marzo 2018, han sido suministrados por la Agencia Estatal de Meteorología (AEMET) en su estación "Cabo Vilan", situada en el mismo parque eólico. Durante el estudio, la zona presentó una alta humedad relativa media anual $(84,5 \%)$, una suave temperatura media anual $\left(14,7^{\circ} \mathrm{C}\right)$ y alta velocidad de precipitación $(1035 \mathrm{~L} /$ $\mathrm{m}^{2}$ año) con 188 días de precipitación anual. Estos valores indican un elevado tiempo de humectación de la superficie de aluminio, lo que ha favorecido el proceso de corrosión atmosférica de este metal. Asimismo, la zona de experimentación ha estado expuesta a fuertes vientos marinos, principalmente en las direcciones NNE Y NE. Ya que las estaciones de ensayo estaban orientadas mirando al norte (N), han estado expuestas a fuertes vientos con un arrastre considerable del aerosol marino (salinidad atmosférica) que ha acelerado el proceso de corrosión atmosférica. En la Fig. 1 se muestra la rosa anual de los vientos de la zona experimental en el año de estudio.

Las probetas de ensayo se expusieron al aire libre formando un ángulo de $90^{\circ}$ con la horizontal

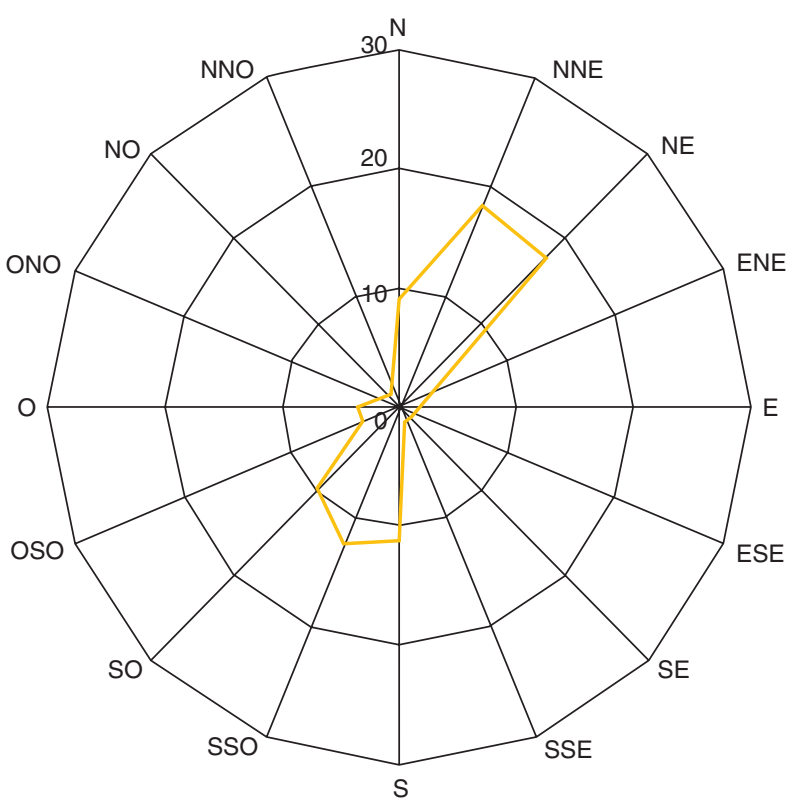

Figura 1. Rosa de vientos correspondiente a la zona de experimentación en el año de estudio (Abril 2017-Marzo 2018).

Tabla 1. Composición química ( $\%$ en peso) del Al 1050 utilizado

\begin{tabular}{lcccccccc}
\hline $\mathbf{F e}$ & $\mathbf{S i}$ & $\mathbf{T i}$ & $\mathbf{Z n}$ & $\mathbf{M n}$ & $\mathbf{M g}$ & $\mathbf{C u}$ & $\mathbf{C r}$ & $\mathbf{A l}$ \\
\hline 0,32 & 0,06 & 0,02 & 0,011 & 0,002 & 0,002 & 0,0004 & 0,0002 & 99,55 \\
\hline
\end{tabular}


para reproducir mejor la posición que las chaspas de aluminio suelen tener cuando se utilizan como recubrimiento de fachadas en edificación. Se expusieron durante un año en seis estaciones de ensayo situadas a diferentes distancias de la línea de costa (Tabla 2) dentro del Parque eólico de Cabo Vilano (Camariñas, Galicia), procediéndose a su retirada después de 3, 6, 9 y 12 meses de exposición. La atmósfera que rodea a las estaciones de ensayo puede considerarse marina pura con muy bajo contenido en el contaminante $\mathrm{SO}_{2}\left(0,7-0,9 \mathrm{mg} / \mathrm{m}^{2}\right.$ día; clasificación P0 de acuerdo a la norma EN ISO 9223 (2012)). La descripción del tipo de estación de ensayo utilizada se encuentra en una publicación anterior (Alcántara et al., 2015). Simultáneamente, se realizaron medidas trimestrales de la velocidad de depósito de cloruros mediante la técnica de la candela húmeda (EN ISO 9225, 2012).

La Fig. 2 muestra la variación de la velocidad anual de depósito de cloruros con la distancia a la costa. Se observa un fuerte descenso de la salinidad atmosférica entre las estaciones de ensayo 1 y 2 (Tabla 2), es decir, las estaciones más próximas a la línea de costa. Un posterior alejamiento respecto a la línea de costa genera un descenso de la salinidad menos acusado.

\subsection{Evaluación del daño por corrosión}

A diferencia de lo que ocurre con el acero, zinc o cobre, en los que la corrosión atmosférica motiva una pérdida generalizada de espesor del material, la corrosión atmosférica del aluminio suele ser de tipo localizado, con formación de picaduras repartidas por toda la superficie metálica, que dejan entre sí amplias áreas de metal intacto. Por ello, en la corrosión atmosférica del aluminio pierde sentido el concepto de penetración media de la corrosión, siendo

TABLA 2. Localización de las estaciones de ensayo, su distancia a la costa en la dirección norte y velocidad anual de depósito de cloruros

\begin{tabular}{|c|c|c|c|}
\hline $\begin{array}{l}\text { Estación de } \\
\text { ensayo }\end{array}$ & $\begin{array}{c}\text { Localización } \\
\text { (coordenadas) }\end{array}$ & $\begin{array}{l}\text { Distancia } \\
\text { a la costa } \\
\text { (m) }\end{array}$ & $\begin{array}{l}\text { Velocidad anual de } \\
\text { depósito de cloruros } \\
\left(\mathrm{mg} / \mathrm{m}^{2} \text { día }\right)\end{array}$ \\
\hline 1 & $\begin{array}{l}43^{\circ} 9 ' 9.80 " \mathrm{~N} \\
9^{\circ} 12^{\prime} 16.71 " \mathrm{O}\end{array}$ & 332 & 1117 \\
\hline 2 & $\begin{array}{l}43^{\circ} 99^{\prime} 4.54 " \mathrm{~N} \\
9^{\circ} 12^{\prime} 13.38^{\prime \prime O}\end{array}$ & 710 & 343 \\
\hline 3 & $\begin{array}{l}43^{\circ} 8,58.12^{\prime \prime} \mathrm{N} \\
9^{\circ} 12^{\prime} 0.13^{\prime \prime} \mathrm{O}\end{array}$ & 820 & 211 \\
\hline 4 & $\begin{array}{l}43^{\circ} 8{ }^{\prime} 46.83 " \mathrm{~N} \\
9^{\circ} 12^{\prime} 1.40^{\prime \prime} \mathrm{O}\end{array}$ & 945 & 258 \\
\hline 5 & $\begin{array}{l}43^{\circ} 8{ }^{\prime} 37.85^{\prime \prime} \mathrm{N} \\
9^{\circ} 11^{\prime} 54.18^{\prime \prime} \mathrm{O}\end{array}$ & 1130 & 80 \\
\hline 6 & $\begin{array}{l}43^{\circ} 8,51.22^{\prime \prime} \mathrm{N} \\
9^{\circ} 10^{\prime} 45.09^{\prime \prime} \mathrm{O}\end{array}$ & 1250 & 92 \\
\hline
\end{tabular}

más adecuado expresar la corrosión como pérdida de masa por unidad de superficie $\left(\right.$ p.ej. $\left.g \cdot \mathrm{m}^{-2}\right)$. Es también importante determinar la frecuencia $\mathrm{y}$, sobre todo, la profundidad de las picaduras y su velocidad de avance, teniendo en cuenta que no son uniformes y presentan una distribución estadística de tamaños (Feliu y Morcillo, 1982). Por ello, en este estudio se ha determinado tanto la pérdida de masa por unidad de superficie $\left(\mathrm{g} \cdot \mathrm{m}^{-2}\right)$ experimentada por el aluminio, como la profundidad de las picaduras existentes.

En el primer caso, la pérdida de masa se realizó por triplicado, eliminando previamente los productos de corrosión con ácido nítrico a $25^{\circ} \mathrm{C}$ (EN ISO 9226, 2012). Sin embargo, a veces las picaduras formadas en la superficie del aluminio no facilitan la eliminación completa de los productos de corrosión formados, ya que en ocasiones siguen caminos tortuosos formando túneles que transcurren por debajo de la superficie no atacada del aluminio (Fig. 3).

En el segundo caso, la estimación de la profundidad de las picaduras se realizó en una serie limitada de probetas de ensayo, en un área de $2 \times 1 \mathrm{~cm}^{2}$ representativa de la superficie de aluminio expuesta a la atmósfera, mediante un perfilómetro confocal interferométrico Sensofar Pl $\mu 2300$. Se realizaron determinaciones en cinco zonas de la superficie de observación. En la Fig. 4 se expone la secuencia experimental seguida con el perfilómetro para estimar la profundidad o penetración de las picaduras.

\subsection{Naturaleza de los productos de corrosión formados}

La caracterización de las fases cristalinas, formadas durante la exposición atmosférica del aluminio, y su evolución con el tiempo de exposición se realizó

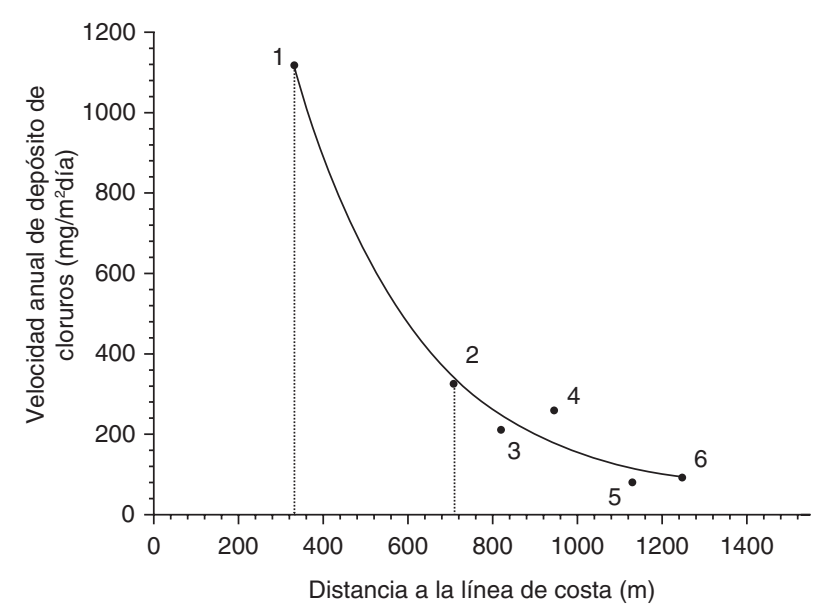

FIGURA 2. Variación de la velocidad anual de depósito de cloruros en las estaciones de ensayo (ver Tabla 2) con la distancia a la costa. 

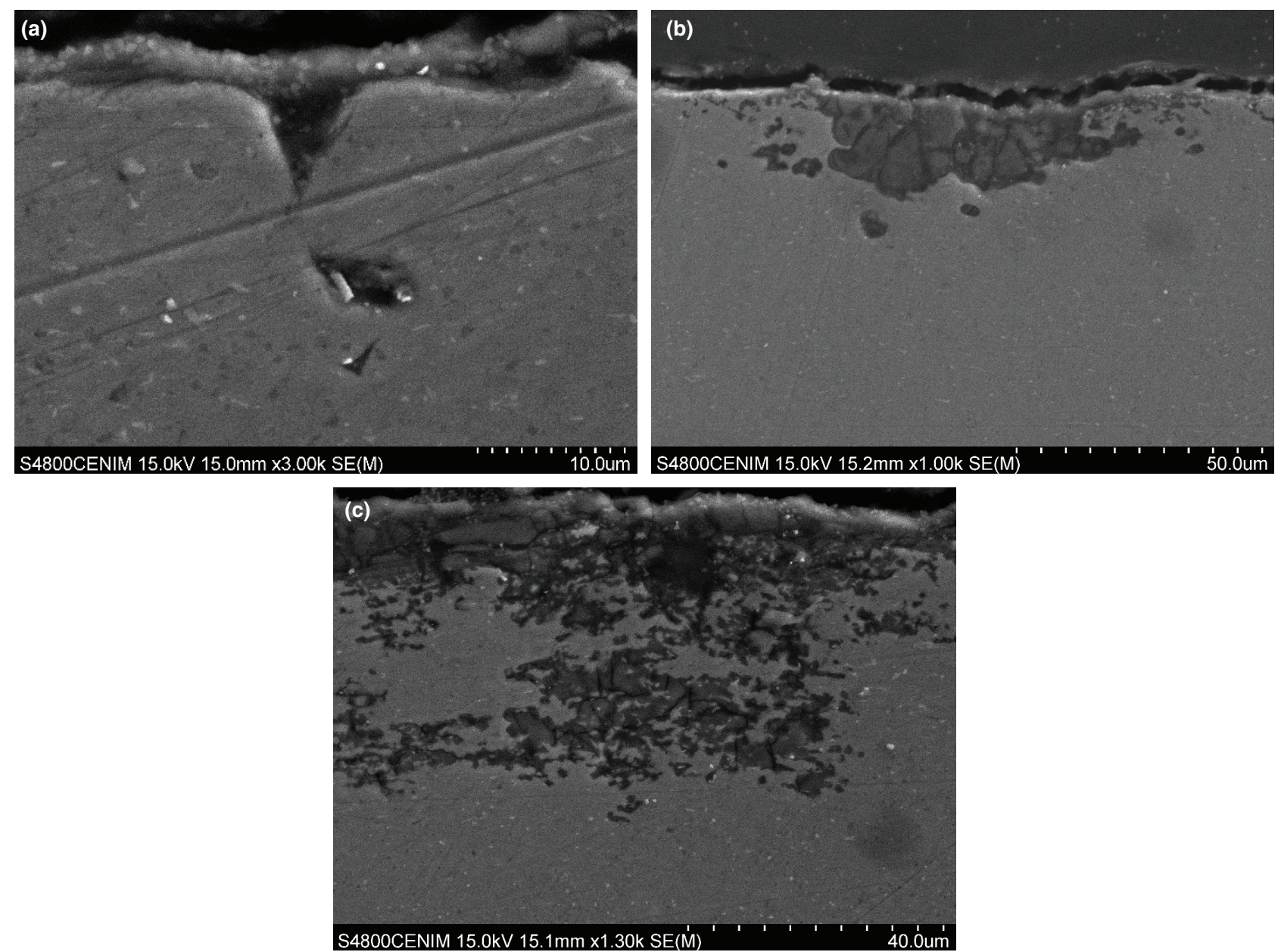

FiguRA 3. Rara vez en el picado del aluminio expuesto a la atmósfera marina se observan picaduras con su morfología clásica (a). Lo más común es que en las secciones transversales realizadas a la superficie del aluminio se observen junto a las zonas de ataque localizado (b), zonas de propagación de ataque correspondientes a picaduras más o menos cercanas a esa sección transversal (c).

a partir de los espectros de difracción de rayos X que se adquirieron con geometría de incidencia rasante. Estas medidas se realizaron en un difractómetro Bruker AXS D8 operado a $40 \mathrm{kV}$ y $30 \mathrm{~mA}$, provisto de un tubo de rayos $\mathrm{X}$ de ánodo de Co y un espejo Goebel para conseguir un haz paralelo de rayos X y eliminar la contribución de la radiación $\mathrm{K}_{\beta}$. Bajo un ángulo de incidencia de $2^{\circ}$, se realizaron barridos en $2 \theta$ entre 6 y $80^{\circ}$ con un paso de $0.03^{\circ}$ y un tiempo de recuento de $4 \mathrm{~s}$ por paso. A partir de los patrones de difracción, se determinaron los compuestos cristalinos presentes con la ayuda de un software de búsqueda y la base de datos cristalográfica JCPDS editada por el ICDD (International Centre for Diffraction Data).

Para la cuantificación de las fases presentes se realizó un ajuste del difractograma por el método Rietveld, recurriendo a la versión 4.2 del paquete informático TOPAS (Bruker AXS) y a la información cristalográfica de la base de datos Pearson de estructuras cristalinas (Villars y Cenzual, 2018).

\subsection{Morfología del ataque experimentado por el aluminio}

La caracterización morfológica del ataque experimentado por el aluminio se realizó con un microscopio electrónico de barrido (MEB) JEOL JSM - $7600 \mathrm{~F}$, equipado con detectores de electrones secundarios y retrodispersados, así como de un sistema de microanálisis Oxford Inca de dispersión de energías (EDS). Las imágenes obtenidas con el microscopio electrónico se realizaron a diferentes aumentos con un voltaje de aceleración de $15 \mathrm{kV}$.

\section{RESULTADOS Y DISCUSIÓN}

A diferencia de otros metales y aleaciones, en los que la acción atmosférica promueve un ataque generalizado de la superficie metálica, en el aluminio y sus aleaciones la corrosión atmosférica da lugar a la formación de picaduras en puntos aislados de la superficie manteniéndose inalterada el resto de 

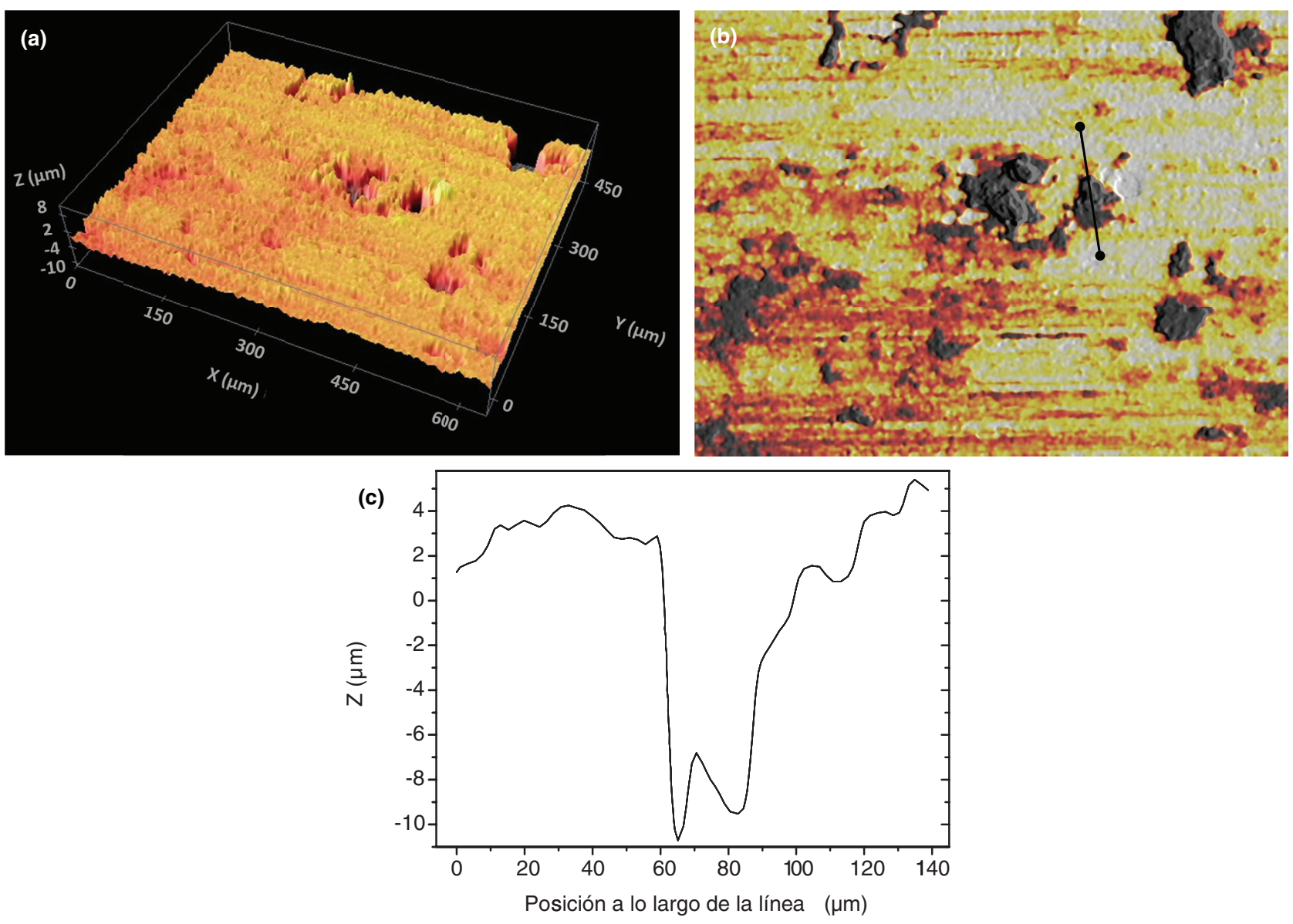

FIgURA 4. Secuencia experimental seguida con el perfilómetro confocal utilizado para la estimación de la profundidad de las picaduras: a) imagen $3 \mathrm{D}, \mathrm{b}$ ) imagen $2 \mathrm{D}$ y c) perfil de la picadura trazada a lo largo de la línea de color negro en la imagen $2 \mathrm{D}$.

TABLA 3. Variación de la velocidad de corrosión (pérdida de masa) del aluminio 1050 con el tiempo de exposición en las distintas estaciones de ensayo

\begin{tabular}{lccccc}
\hline \multirow{2}{*}{$\begin{array}{l}\text { Estación de ensayo } \\
\text { (ver Tabla 2) }\end{array}$} & $\begin{array}{c}\text { Velocidad anual de depósito } \\
\text { de cloruros (mg/m² día) }\end{array}$ & $\mathbf{3}$ meses & $\mathbf{6}$ meses & $\mathbf{9}$ meses & $\mathbf{1 2}$ meses \\
\hline 1 & 1117 & $18,20 \pm 0,46$ & $13,84 \pm 0,40$ & $9,78 \pm 0,43$ & $9,61 \pm 0,58$ \\
2 & 343 & $15,79 \pm 4,17$ & $10,44 \pm 1,80$ & $7,14 \pm 0,30$ & $8,33 \pm 0,52$ \\
3 & 211 & $10,24 \pm 1,83$ & $10,27 \pm 0,09$ & $9,16 \pm 0,57$ & $6,60 \pm 0,75$ \\
4 & 258 & $12,16 \pm 2,87$ & $11,58 \pm 1,08$ & $7,25 \pm 1,44$ & $7,50 \pm 0,47$ \\
5 & 80 & $8,64 \pm 0,00$ & $8,21 \pm 1,27$ & $7,76 \pm 0,65$ & $6,96 \pm 0,27$ \\
6 & 92 & $8,79 \pm 0,92$ & $9,54 \pm 0,07$ & $5,61 \pm 0,66$ & $4,15 \pm 0,05$ \\
\hline
\end{tabular}

la superficie metálica. Las picaduras van progresando hacia el interior del metal conforme avanza el tiempo de exposición.

La Tabla 3 muestra las velocidades de corrosión del aluminio 1050 y su evolución con el tiempo de exposición en las distintas estaciones de ensayo. La interacción del aluminio con la película acuosa existente sobre la superficie metálica (humedad adsorbida, capa visible de agua, etc.), que contiene especies cloruradas procedentes del aerosol marino, acelera la corrosión de este metal. La velocidad de corrosión del aluminio disminuye conforme nos alejamos de la línea de costa (Fig. 5), al disminuir paralelamente la salinidad atmosférica del lugar (Fig. 2). Asimismo, la velocidad de corrosión del aluminio disminuye con el tiempo de exposición (Fig. 6) por la existencia de productos de corrosión en el seno de las picaduras que dificultan la entrada de las especies agresivas del medio $\left(\mathrm{O}_{2}, \mathrm{H}_{2} \mathrm{O}, \mathrm{Cl}^{-}\right.$, $\mathrm{SO}_{4}{ }^{2-}$, etc.) hacia el sustrato metálico. 
En cuanto a las estimaciones realizadas sobre la profundidad de las picaduras formadas sobre el aluminio en diferentes condiciones de exposición (Tabla 4), puede observarse claramente cómo aumenta la profundidad máxima y media con el tiempo de exposición. No obstante, el avance de

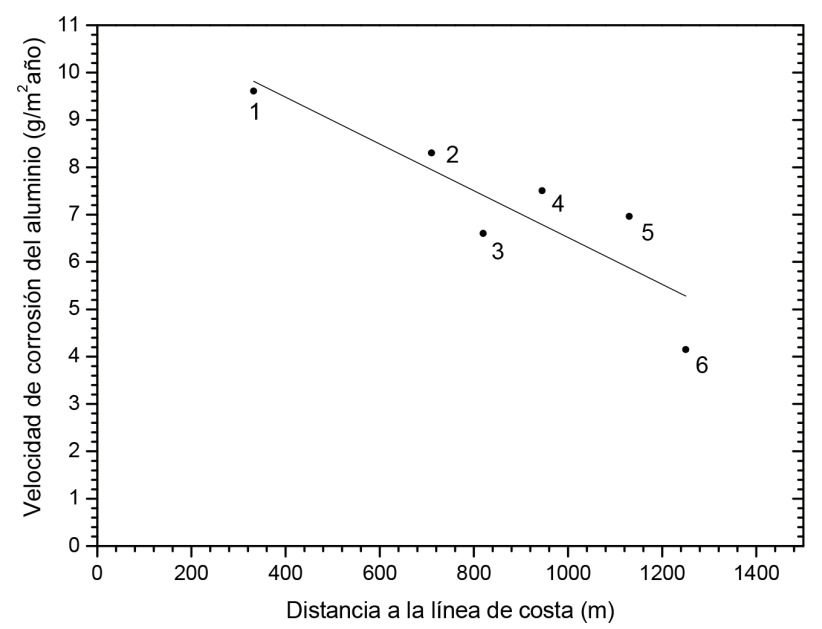

FIGURA 5. Variación de la velocidad de corrosión anual del aluminio con la distancia a la costa (los números corresponden a las estaciones de ensayo, Tabla 2).

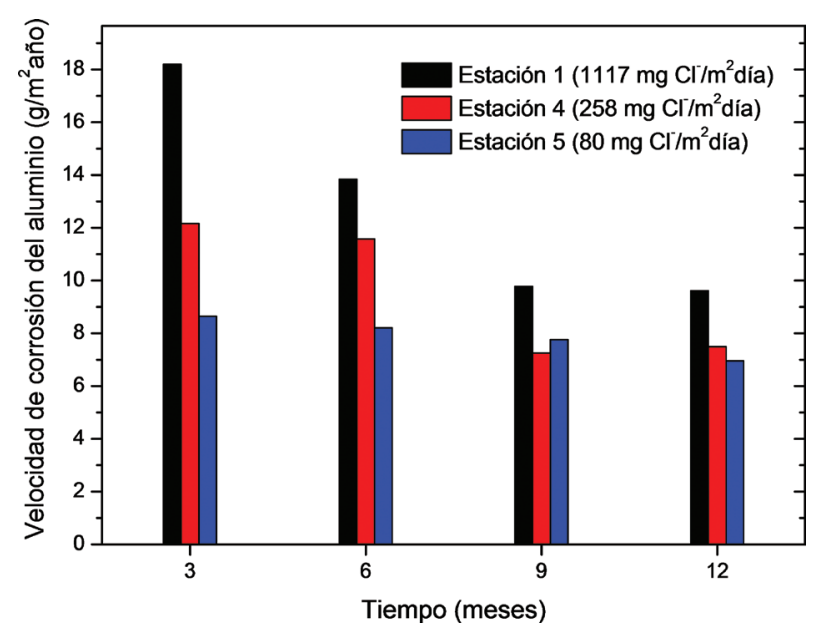

Figura 6. Variación de la velocidad de corrosión del aluminio con el tiempo de exposición. las picaduras parece amortiguarse relativamente pronto conforme avanza el tiempo de exposición, de acuerdo con lo observado también por otros investigadores (Mattsson y Lindgren, 1968; Otero et al., 1971; Kentzler y Hoff, 1975; Otero et al., 1978), de tal modo que un aumento de 3 a 12 meses del tiempo de exposición en las condiciones de mayor y menor velocidad de depósito de cloruros en la atmósfera, tan solo logra aumentar la profundidad media de las picaduras en un 19 y $39 \%$ respectivamente.

Otro hecho que conviene resaltar es el reducido efecto de la salinidad atmosférica en la magnitud de los parámetros de profundidad anual de las picaduras. Un aumento muy elevado en la velocidad de depósito de cloruros de la atmósfera, de $92 \mathrm{mg} / \mathrm{m}^{2}$ día (estación 6) a $1117 \mathrm{mg} / \mathrm{m}^{2}$ día (estación 1), unas 12 veces superior, tan solo ha supuesto un incremento aproximado del $87 \%$ y $56 \%$ en la profundidad máxima y media de las picaduras respectivamente. Tanto este hecho como el anterior, confirman que la existencia de los productos de corrosión en el interior de las picaduras dificulta enormemente la entrada de los iones $\mathrm{Cl}^{-}$y $\mathrm{SO}_{4}{ }^{2-}$ a su través para interaccionar con el sustrato metálico.

Los patrones de difracción de rayos $\mathrm{X}$ obtenidos por ángulo rasante, de acuerdo con la base cristalográfica JCDPDS, indicaron la presencia mayoritaria de aluminio y del compuesto $\mathrm{Al}(\mathrm{OH})_{3}$ (minoritaria). Hay que tener presente que en un gran porcentaje de la superficie del aluminio no se forman picaduras. No ha resultado fácil establecer cuál de los hidróxidos cristalinos con esta estequiometría (gibsita, bayerita, nordstandita o doylita) está presente ya que, como se muestra en la Tabla 5, sus reflexiones más intensas se encuentran en posiciones muy

TABla 5. Posición de las reflexiones más intensas de los hidróxidos de $\mathrm{Al}$ para la radiación de $\mathrm{Co}$

\begin{tabular}{lccc}
\hline Phase & $\begin{array}{c}\text { Archivo } \\
\text { JCPDS }\end{array}$ & $\begin{array}{c}\text { Reflexión } \\
\text { (hkl) }\end{array}$ & $\begin{array}{c}\text { Posición del } \\
\text { pico (2) })\end{array}$ \\
\hline Gibsita & $033-0018$ & $(002) /(110)$ & $21,262 / 23,616$ \\
Nordestrandita & $024-0006$ & $(010) /(001)$ & $21,525 / 23,900$ \\
Bayerita & $020-0011$ & $(001)(020)$ & $21,895 / 23,733$ \\
Doylita & $038-0376$ & $(010) /(10-1)$ & $21,507 / 24,035$ \\
\hline
\end{tabular}

TABLA 4. Estimaciones sobre la profundidad de las picaduras formadas sobre el aluminio en diferentes condiciones de exposición

\begin{tabular}{|c|c|c|c|c|c|c|}
\hline \multirow{2}{*}{$\begin{array}{l}\text { Estación de } \\
\text { ensayo } \\
\text { (ver Tabla 2) }\end{array}$} & \multirow{2}{*}{$\begin{array}{c}\text { Tiempo de } \\
\text { exposición (meses) }\end{array}$} & \multirow{2}{*}{$\begin{array}{l}\text { Velocidad anual de depósito } \\
\text { de cloruros ( } \mathrm{mg} / \mathrm{m}^{2} \text { día) }\end{array}$} & \multirow{2}{*}{$\begin{array}{c}\text { Número de } \\
\text { observaciones }\end{array}$} & \multicolumn{3}{|c|}{ Profundidad de las picaduras $(\mu \mathrm{m})$} \\
\hline & & & & mínima & máxima & media \\
\hline \multirow[t]{2}{*}{1} & 3 & 950 & 14 & 2 & 22 & 8,4 \\
\hline & 12 & 1117 & 27 & 3 & 30 & 10,0 \\
\hline \multirow[t]{2}{*}{6} & 3 & 29 & 8 & 3 & 10 & 4,6 \\
\hline & 12 & 92 & 16 & 3 & 16 & 6,4 \\
\hline
\end{tabular}


cercanas. Para solucionar este problema, se recurrió al método Rietveld, el cual permite el ajuste simultaneo de todas las reflexiones y determinar con cuál de los posibles hidróxidos de aluminio se obtiene un mejor ajuste del difractograma, resultando ser el compuesto gibsita, lo que supone una novedad en la literatura científica sobre la corrosión atmosférica del aluminio en ambientes ricos en iones cloruro. En la Fig. 7 se expone la representación típica de los difractogramas de rayos $\mathrm{X}$ obtenidos.

La aplicación del refinamiento por Rietveld permitió, asimismo, conocer los porcentajes de la superficie de aluminio recubierta por gibsita en las diferentes condiciones de exposición (Tabla 6). Puede observarse cómo este porcentaje aumenta con la salinidad de la atmósfera y tiempo de exposición.

La Fig. 8 muestra las micrografías MEB de la superficie del aluminio después de 3 meses de exposición en distintas condiciones ambientales. Puede observarse cómo en la estación de ensayo más alejada de la línea de costa, donde menor es la velocidad de depósito de cloruro, una gran parte de la superficie no está recubierta de productos de corrosión (Fig. 8a). En cambio, en la estación más cercana a la

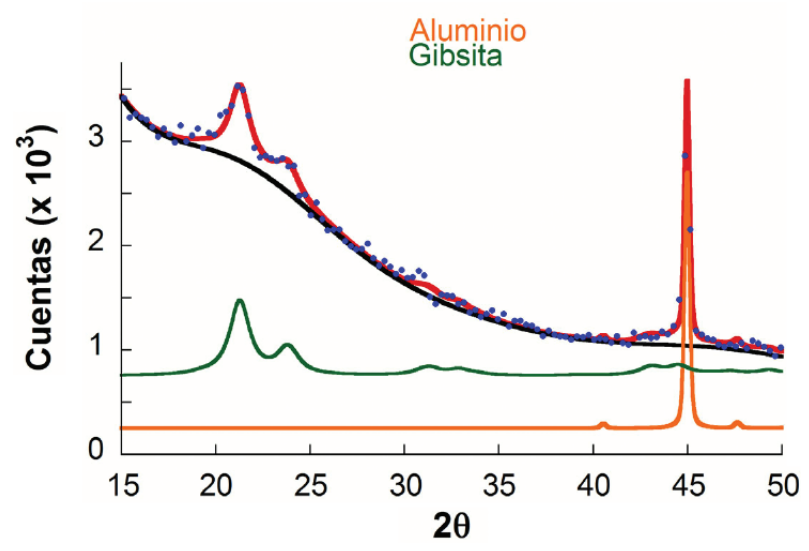

FIGURA 7. Representación típica para la comparación de los patrones de difracción de rayos $\mathrm{X}$, observado (círculos azules) y calculado ajustado por el método Rietveld (línea roja continua), correspondientes a los productos de corrosión formados sobre el aluminio expuesto a las atmósferas marinas de Cabo vilano. La contribución del aluminio base y de la gibsita se muestra en colores naranja y verde respectivamente. línea de costa, donde mayor es la velocidad de depósito de cloruro, la superficie del aluminio está prácticamente cubierta de productos de corrosión (Fig. $8 b)$ procedentes de picaduras más o menos cercanas que se van extendiendo por el resto de la superficie metálica. Se detecta, mediante microanálisis EDS (Fig. 8c), la presencia de $\mathrm{O}$ y $\mathrm{Al}$, correspondientes a los productos de corrosión formados, así como $\mathrm{Cl}$ y $\mathrm{Na}$ procedentes del aerosol marino depositado sobre ellos.

La observación MEB de cortes transversales de las muestras de aluminio puede mostrar la formación de picaduras. A modo de ejemplo, la Fig. 9a muestra la formación de una de esas picaduras, en la que también pueden observarse zonas de ataque del aluminio que corresponden a cortes transversales en los caminos de propagación de otras picaduras cercanas.

Interesa conocer la composición de los productos de corrosión en distintas zonas en el seno de la picadura. El análisis EDS (Fig. 9b) detecta la presencia de $\mathrm{Cl}, \mathrm{S}$ y de $\mathrm{Na}$ y $\mathrm{Mg}$. Todos ellos, en su forma iónica, forman parte del aerosol marino (Pilson, 1998). Puede observarse cómo los aniones $\mathrm{Cl}^{-}$y $\mathrm{SO}_{4}{ }^{2-}$ aumentan su concentración conforme nos adentramos en el seno de la picadura, ya que migran hacia el fondo de la misma (Santamaría, 1990) para neutralizar el exceso de carga positiva de la zona anódica.

\section{CONCLUSIONES}

El estudio llevado a cabo sobre corrosión atmosférica del aluminio 1050 en atmósferas costeras ha conducido a las siguientes conclusiones:

- El alcance de la velocidad de corrosión atmosférica del aluminio disminuye conforme nos alejamos de la línea de costa.

Un aumento sostenible de la velocidad de depósito de cloruro de la atmósfera marina no se traduce en un aumento del mismo orden en la velocidad de corrosión del aluminio, debido a la existencia de productos de corrosión en el interior de las picaduras que dificultan enormemente la entrada de los iones $\mathrm{Cl}^{-}$a su través para interaccionar con el sustrato metálico.

TABla 6. Porcentajes de la superficie del aluminio 1050 cubierta por Gibsita $\left(\mathrm{Al}(\mathrm{OH})_{3}\right)$ en diferentes condiciones de exposición. Información obtenida a partir de los difractogramas de DRX ajustados por el método Rietveld

\begin{tabular}{|c|c|c|c|}
\hline $\begin{array}{l}\text { Estación de ensayo } \\
\text { (ver Tabla 2) }\end{array}$ & $\begin{array}{l}\text { Velocidad anual de depósito } \\
\text { de cloruros ( } \mathrm{mg} / \mathrm{m}^{2} \text { día) }\end{array}$ & $\begin{array}{c}\text { Tiempo de exposición } \\
\text { (meses) }\end{array}$ & $\begin{array}{c}\text { Porcentaje } \\
(\%)\end{array}$ \\
\hline \multirow[t]{2}{*}{1} & 950 & 3 & 5,8 \\
\hline & 1117 & 12 & 31,2 \\
\hline \multirow[t]{2}{*}{6} & 29 & 3 & 0 \\
\hline & 92 & 12 & 4,6 \\
\hline
\end{tabular}



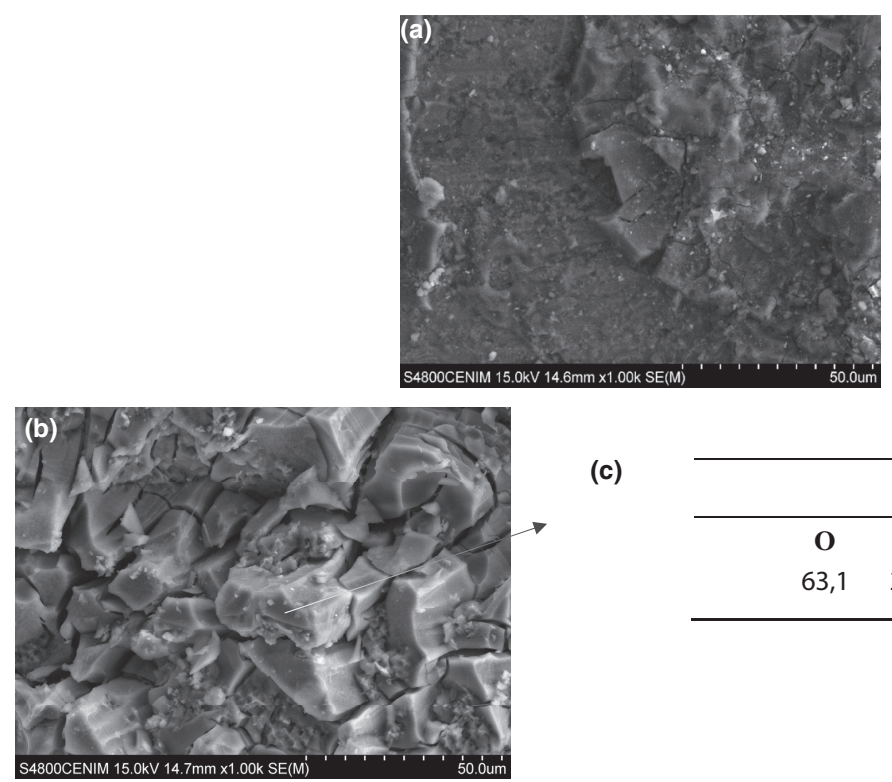

(c)

\begin{tabular}{cccc}
\hline \multicolumn{4}{c}{$\%$ en peso } \\
\hline $\mathbf{O}$ & Al & Cl & Na \\
63,1 & 26,1 & 9,8 & 1 \\
\hline
\end{tabular}

FIgURA 8. Caracterización mediante MEB/EDS de la superficie de probetas de aluminio 1050 expuestas durante 3 meses en las atmósferas de menor (a) y mayor (b, c) velocidad de depósito de cloruros.

(a)

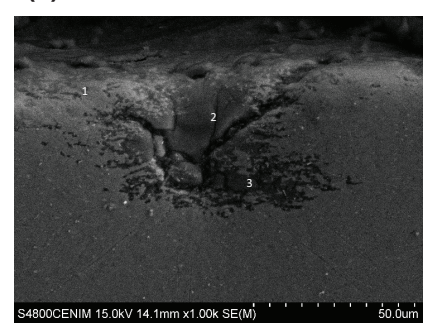

(b)

\begin{tabular}{|c|c|c|c|c|c|}
\hline \multirow{2}{*}{ Zona } & \multicolumn{5}{|c|}{ \% en peso } \\
\cline { 2 - 6 } & $\mathbf{O}$ & Al & Cl & S & Na + Mg \\
\hline 1 & 59,3 & 39,0 & 0,4 & 0,7 & 0,6 \\
\hline 2 & 81,1 & 15,7 & 0,8 & 1,2 & 1,2 \\
\hline 3 & 79,8 & 17,1 & 0,7 & 1,3 & 1,1 \\
\hline
\end{tabular}

FIGURA 9. Formación de una picadura en la superficie del aluminio 1050. Observación realizada por MEB (a) y resultados del microanálisis de rayos $\mathrm{X}$ por dispersión de energías (EDS) en distintas zonas (b).

- Tanto la corrosión del aluminio como la profundidad de las picaduras formadas se amortigua notablemente conforme avanza el tiempo de exposición.

- El producto de corrosión formado sobre el aluminio en estos ambientes corresponde al hidróxido de aluminio $\left(\mathrm{Al}(\mathrm{OH})_{3}\right)$, tipo gibsita.

- El progreso en la penetración de la picadura en el sustrato metálico se debe a la presencia de los aniones $\mathrm{Cl}^{-}$y $\mathrm{SO}_{4}^{2-}$, procedentes del aerosol marino, que migran hacia el fondo de la picadura (zona anódica).

\section{AGRADECIMIENTOS}

Los autores quieren agradecer a las empresas ENEL y GAS NATURAL-FENOSA por permitir localizar las estaciones de ensayo en sus instalaciones del Parque eólico de Cabo Vilano (Camariñas, España). Además, también quieren agradecer a la agencia española de meteorología
(AEMET) por los datos proporcionados sobre parámetros meteorológicos en el área de estudio, así como a la Dra. Ana Conde por su ayuda en el manejo del perfilómetro confocal perteneciente al grupo COPROMAT del Centro Nacional de Investigaciones Metalúrgicas.

\section{REFERENCES}

Alcántara, J., Chico, B., Díaz, I., de la Fuente, D., Morcillo, M. (2015). Airborne chloride deposit and its effect on marine atmospheric corrosion of mild steel. Corros. Sci. 97, 74-88. https://doi.org/10.1016/j.corsci.2015.04.015.

Aziz, P.M., Godard, H.P. (1952). Pitting Corrosion Characteristics of Aluminum - Influence of Magnesium and Manganese. Ind. Eng. Chem. 44 (8), 1791-1795. https://doi. org/10.1021/ie50512a028.

Berukshtis, G.K., Klark, G.B. (1966). Atmospheric corrosion of steel, zinc, cadmium, copper and aluminium in different coastal and continental regions. In Corrosion of metals and alloys, Tomashov, N.D. and Mirolyubev, E.N., Editors, Jerusalem, Israel pp. 281-297.

Booth, F.F., Godard, H.P. (1965). Corrosion Behavior of Aluminum. Alloys in Seawater, Congres international de la corrosion marine et des salissures, Cannes. 
Carter, V.E. (1968). Atmospheric corrosion of Aluminum and Its Alloys: Results of six-year exposure tests. En: Metal corrosion in the atmosphere. ASTM STP 435, American Society for Testing and Materials, pp. 257-270.

EN ISO 9226 (2012). Corrosion of metals and alloys, corrosivity of atmospheres, determination of corrosion rate of standard specimens for the evaluation of corrosivity. Anexo A, European Committee for Standardization, Brussels.

EN ISO 9225 (2012). Corrosion of metals and alloys, corrosivity of atmospheres, measurement of environmental parameters affecting corrosivity of atmospheres. European Committee for Standardization, Brussels.

EN ISO 9223 (2012). Corrosion of metals and alloys, corrosivity of atmospheres, clasification, determination and estimation. European Committee for Standardization, Brussels.

de la Fuente, D., Otero-Huerta, E., Morcillo, M. (2007). Studies of long-term weathering of aluminium in the atmosphere. Corros. Sci. 49 (7), 3134-3148. https://doi.org/10.1016/j. corsci.2007.01.006.

Feliu, S., Morcillo, M. (1982). Corrosión y protección de los metales en la atmósfera. Edicions Bellaterra, S.A., Barcelona, España.

Golubev, A.I., Kadyrov, M.K. (1969). Calculation of moistening and metallic corrosion in atmospheric environment. Paper presented at the Third international Congress on Metallic Corrosion, Moscow.

González, J.A., Morcillo, M., Escudero, E., López, V., Otero, E. (2002). Atmospheric corrosion of bare and anodized aluminium in a wide range of environmental conditions. Part I: Visual observations and gravimetric results. Surf. Coat. Tech. 153 (2-3), 225-234. https://doi.org/10.1016/ S0257-8972(01)01680-2

Graedel, T.E. (1989). Corrosion mechanisms for aluminum exposed to the atmosphere. J. Electrochem. Soc. 136 (4), 204C-212C. https://doi.org/10.1149/1.2096869.

Haynie, F.H., Spence, J.W., Upham, J.B. (1976). Effects of gaseous pollutants on materials : a chamber study. Report EPA-600/3-76-015. U.S. Environmental Protection Agency, Research Triangle Park, USA.

Kaesche, H. (1974). Localized corrosion. NACE, Williamsburg Conference, Houston, p. 516.

Kentzler, O., Hoff, P. (1975). Behavior of an Aluminum Roof Exposed Over a Prolonged Period to Industrial Atmosphere. Schweiz. Alum. Rundsch. 25 (1), 11-19.

Kucera, V., Mattson, E. (1987). Atmospheric Corrosion. In Corrosion Mechanisms. Mansfeld, F. (Ed.), Dekker, New York.

Lashermes, M., Guilhaudis, A., Reboul, M., Trentelivres, G. (1982). Thirty-year atmospheric corrosion of aluminium alloys in France. In Atmospheric Corrosion, W. H. Ailor (Ed.), John Wiley and Sons, New York, pp. 353-364.

Leygraf, C., Wallinder, I.O., Tidblad, J., Graedel, T. (2016). Atmospheric corrosion. $2^{\text {nd }}$ Edition, John Wiley \& Sons, Inc., USA.

Liang, M., Melchers, R., Chaves, I. (2018). Corrosion and pitting of 6060 series aluminium after 2 years exposure in seawater splash, tidal and immersion zones Corros Sci. 140, 286-296. https://doi.org/10.1016/j.corsci.2018.05.036.

Liu, Y., Wang, Z., Ke, W. (2014). Study on influence of native oxide and corrosion products on atmospheric corrosion of pure Al. Corros. Sci. 80, 169-176. https://doi.org/10.1016/j. corsci.2013.11.027.

Longo, F.N., Durmann, G.J. (1978). Atmospheric factors affecting the corrosion of engineering metals. ASTM STP 646,
ASTM International, West Conshohocken, PA, USA, pp. 97-114.

McGeary, F.L., Summerson, T.J., Ailor, W.H. (1968). Atmospheric Exposure of Nonferrous Metals and Alloys-Aluminum Seven-year Data. En: Metal corrosion in the atmosphere. ASTM STP 435, American Society for Testing and Materials, pp. $141-174$.

Mikhailovskii, Y.N., Klark, G.B., Shuvakhina, L.A., Agafonov, V.V., Zhuravlena, N.I. (1973). Calculation of atmospheric corrosion rate of aluminium and its alloys in various climatic zones according to meteorological parameters. Prot. Met. 9 (3), 240-246.

Morcillo, M., Almeida, E., Rosales, B. (2000a). Corrosion of Aluminium in pure Marine Atmospheres. Aluminium 76 $(7 / 8), 610-615$.

Morcillo, M. Almeida, E., Rosales, B. (2000b). Corrosion of Aluminium in $\mathrm{SO}_{2}$ - Polluted Marine Atmospheres. Aluminium 76 (12), 1066-1070.

Morcillo, M., Almeida, E., Rosales, B. (2000c). Degradation of Aluminium in Rural Atmospheres. Aluminium 76 (4), 316-319.

Natesan, M., Venkatachari, G., Palaniswamy, N. (2006). Kinetics of atmospheric corrosion of mild steel, zinc, galvanized iron and aluminium at 10 exposure stations in India. Corros. Sci. 48 (11), 3584-3608. https://doi.org/10.1016/j. corsci.2006.02.006.

Otero, E., Lizarbe, R., Feliu, S. (1971). Comportamiento de probetas pulidas de aluminio expuestas en un atmósfera marina de España. Rev. Metal. 7 (5), 359-368.

Otero, E., Lizarbe, R., Feliu, S. (1978). Behaviour of aluminium during 10 years' exposure at a coastal test site at Alicante. Brit. Corros. J. 13 (2), 82-84. https://doi. org/10.1179/000705978798318800.

Pilson, M.E.Q. (1998). An Introduction to the Chemistry of the Sea. First Edition, Ed., Pearson Education Inc., Prentice Hall, UK

Portella, M.O.G., Portella, K.F., Pereira, P.A.M., Inone, P.C., Brambilla, K.J.C., Cabussú, M.S., Cerqueira, D.P., Salles, R.N. (2012). Atmospheric corrosion rates of copper, galvanized steel, carbon steel and aluminum in the metropolitan region of Salvador, BA, Northeast Brazil. Procedia Engineering 42, 171-185. https://doi.org/10.1016/j.proeng.2012.07.408.

Santamaría, A. (1990). Corrosión del aluminio 1050 en el País Vasco, Ph.D. Thesis, Universidad del País Vasco.

Speding, P.L. (1971). Corrosion by atmospheric sulphur dioxide. Australas. Corros. Eng. 15 (8), 27-36.

Tidblad, J., Kucera, V., Mikhailov, A.A. (1998). Statistical analysis of 8 year materials exposure and acceptable deterioration and pollution levels. Swedish Corrosion Institute, Stockholm, Sweden.

Vargel, C. (1981). El comportamiento del aluminio y sus aleaciones. Urmo S.A. de Ediciones, España.

Vera, R., Delgado, D., Rosales, B.M. (2006). Effect of atmospheric pollutants on the corrosion of high power electrical conductors: Part 1. Aluminium and AA6201 alloy. Corros. Sci. 48 (10), 2882-2900. https://doi.org/10.1016/j.corsci.2005.11.012.

Villars, P., Cenzual, K. (2018). Pearson's Crystal Structure Database for Inorganic Compounds. Release 2017/2018, ASM International, Materials Park, Ohio, USA.

Walton, C.J., Sprowls, D.O., Nock, J.A. (1953). Resistance of aluminium alloys to weathering. Corrosion 9 (10), 345-358. https://doi.org/10.5006/0010-9312-9.10.345.

Walton, S.I., King, W. (1955). Symposium on Atmospheric Corrosion of Non-ferrous Metals, American Society for Testing Materials, PA, USA. 